\title{
Random field interferometry for medical ultrasound
}

\section{Conference Paper}

Author(s):

Ulrich, Ines E.; Boehm, Christian; Fichtner, Andreas

Publication date:

2020-03

Permanent link:

https://doi.org/10.3929/ethz-b-000432775

Rights / license:

In Copyright - Non-Commercial Use Permitted

Originally published in:

Proceedings of SPIE 11319, https://doi.org/10.1117/12.2559852 


\title{
Random field interferometry for medical ultrasound
}

\author{
Ines Elisa Ulrich, Christian Boehm and Andreas Fichtner \\ Department of Earth Sciences, ETH Zürich, Sonneggstrasse 5, CH-8092 Zürich, Switzerland
}

\begin{abstract}
We present a novel approach to obtain time-of-flight measurements between transducer pairs in an Ultrasound computed tomography (USCT) scanner by applying the interferometry principle, which has been used successfully in seismic imaging to recover the subsurface velocity structure from ambient noise recordings. To apply this approach to a USCT aperture, random wavefields are generated by activating the emitting transducers in a random sequence. By correlating the random signals recorded by the receiving transducers, we obtain an approximation of the Green's functions between all receiver pairs, where one is acting as a virtual source. This eliminates specific source imprints, and thus avoids the need for reference measurements and calibration. The retrieved Green's functions between any two measurement locations can then be used as new data to invert the sound speed map. On the basis of the cross-correlation travel times a ray-based time-of-flight tomography is developed and solved with an iterative least-squares method. As a proof of concept, the algorithm is tested on numerical breast phantoms in a synthetic 2D study.
\end{abstract}

Keywords: Random field interferometry, ultrasound computed tomography, time-of-flight inversion

\section{INTRODUCTION}

Ultrasound computed tomography (USCT) is frequently used for medical purposes to image soft tissue body parts, as for instance the breast. The property of interest is the speed of sound of the breast tissue, tissue density or attenuation. Commonly, malignant cell regions are denser and stiffer than benign regions, thus speed of sound maps image breast tissue. ${ }^{1-3}$ Breast cancer detection using USCT usually works with a collection of ultrasound scans that measure the pressure wavefield emitted by individual transducers. A state-of-the-art USCT acquisition process often requires a large number of emitter-receiver pairs to obtain a good coverage of the domain of interest and careful calibration of the emitting transducers using reference measurements in water. $^{4-6}$ This is a time-consuming process during which patient movements can degrade the quality of the aquired data. Furthermore, calibration of emitters using reference measurements in water is often subject to strong assumptions, for instance, that the temperature of the water is uniform and constant, which are difficult to establish during real-life aquisition. In recent years, scientific contributions from geophysical research have proven to be a fruitful addition to the medical imaging community. Although the imaged medium in the geophysical application compared to the medical application seems considerably different, Pratt et al. ${ }^{7,8}$ have shown the possibilities arising from applying geophysical imaging methods to breast cancer screening. Further studies, extending the research to image other human body parts such as the brain ${ }^{9}$ or the limbs, ${ }^{10}$ have demonstrated the potential of ultrasound imaging.

With this work, we propose to circumvent the aforementioned challenges of standard USCT data acquisition techniques by transferring yet another theory widely applied in seismic imaging, to breast imaging with USCT. This theory allows us to eliminate the need for calibration and individual source wavefields by implementing the random field interferometry principle in which active sources are substituted by virtual ones without specific imprints. A similar approach of evaluating tissue parameters has been studied by Gallot et al. ${ }^{11}$ and by Sebra et al. ${ }^{12}$ for elastography, where shear waves are used in contrast to pressure waves as considered in this study. In Earth science, random field interferometry has spawned new possibilities to image the interior of the Earth

Further author information: (Send correspondence to I.E.U.)

I.E.U.: E-mail: ines.ulrich@erdw.ethz.ch

C.B.: E-mail: christian.boehm@erdw.ethz.ch

A.F.: E-mail: andreas.fichtner@erdw.ethz.ch 
by using passive measurements of quasi-random wavefields, ${ }^{13,14}$ which has inspired the term ambient noise interferometry. Instead of using the direct transmission data from one source to one receiver, an approximation of the Green's function between any two points can be obtained by cross-correlating recordings of a random wavefield at these two positions ${ }^{15}$ resulting in deterministic signals that constrain the medium.

Cross-correlation is a statistical method to extract the similarities of recordings at two locations as a function of time, suppressing all non-coherent parts in the two signals. Therefore, through constructive interference, the time of flight between two receivers is marked by a peak in the cross-correlation function of two displacement recordings of a random wavefield. The description of seismic and medical imaging problems is based on the same mathematical relations, which allows us to transfer ideas developed in seismic research and apply them in a medical context. In seismic imaging, one relies on ambient random wavefields, generated for example by ocean waves. Since there is no ambient random wavefield in the medical application, we generate a random wavefield actively by a random superposition of individual sources.

As a proof of concept, we develop an algorithm that enables an active generation of a random wavefield and implements the cross-correlation method on the scale of the USCT setup to extract times of flight. We test the algorithm on a 2D numerical breast phantom, representing a coronal slice through the breast. Variations in the speed of sound inside the phantom are included to model regions with malignant cells. The implemented speed of sound values are based on real human soft tissue values reported in the literature ${ }^{1-3}$ with variations on the order of $5 \%$ with respect to the speed of sound of water. To evaluate the quality of the extracted cross-correlation travel times, a ray-based time-of-flight tomography is developed and solved with an iterative least-squares scheme. We show that the travel time shifts between the obtained cross-correlation travel times and the analytical solution for water are accurate enough such that the speed of sound distribution within the phantom can be reconstructed.

\section{METHOD}

\subsection{Random field interferometry}

The propagation of acoustic energy in an inhomogeneous medium is described by the acoustic wave equation that is given in the frequency domain by

$$
-\frac{\omega^{2}}{\rho(\boldsymbol{x}) c^{2}} p(\boldsymbol{x}, \omega)-\partial_{x_{i}}\left(\frac{1}{\rho(\boldsymbol{x})} \partial_{x_{i}} p(\boldsymbol{x}, \omega)\right)=f(\boldsymbol{x}, \omega),
$$

where $\omega$ is the angular frequency, $\rho$ is the density, $c$ is the acoustic velocity and $p(\boldsymbol{x})$ and $f(\boldsymbol{x})$ are the spacefrequency domain representations of the acoustic pressure and the source distribution. We use Einstein's summation convention to sum over repeated lower case subscripts. Equation (1) states that the acoustic pressure at any point $\boldsymbol{x}$ inside the medium is directly connected to the speed of sound of the propagation medium. This is the basic argument for the following analysis on the cross-correlation function of pressure measurements from a random wavefield.

Just as in medical imaging, seismology is often concerned with finding the velocity of a wave traveling through an unknown medium in order to deduce its properties. For convenience, we use the frequency-domain representation of the wavefields and the frequency-domain Green's function $G\left(\boldsymbol{x}, \boldsymbol{x}^{\prime}, \omega\right)$ in the following and omit the dependency on $\omega$. The Green's function is the solution to the acoustic wave equation (1) for a point source at location $\boldsymbol{x}^{\prime}$, therefore satisfying

$$
\mathcal{L}\left[G\left(\boldsymbol{x}, \boldsymbol{x}^{\prime}\right)\right]=\delta\left(\boldsymbol{x}-\boldsymbol{x}^{\prime}\right),
$$

where $\mathcal{L}$ is the forward wave operator collecting the terms $-\frac{\omega^{2}}{\rho(\boldsymbol{x}) c^{2}}(\cdot)-\partial_{x_{i}}\left(\frac{1}{\rho(\boldsymbol{x})} \partial_{x_{i}}(\cdot)\right)$ in equation (1). A relation between the pressure wavefield and its sources can be established by invoking the acoustic frequency-domain representation theorem ${ }^{16,17}$ of the form

$$
p(\boldsymbol{x})=\int_{D} G\left(\boldsymbol{x}, \boldsymbol{x}^{\prime}\right) f\left(\boldsymbol{x}^{\prime}\right) d \boldsymbol{x}^{\prime} .
$$


With the help of the Green's function $G\left(\boldsymbol{x}, \boldsymbol{x}^{\prime}\right)$, the pressure $p$ recorded at position $\boldsymbol{x}$ can be computed for arbitrary sources $f$ located inside the domain $D$ of interest. Two sources at neighbouring positions $\boldsymbol{x}^{\prime}$ and $\boldsymbol{x}^{\prime \prime}$ are assumed to be uncorrelated in time, such that

$$
f^{*}\left(\boldsymbol{x}^{\prime}\right) f\left(\boldsymbol{x}^{\prime \prime}\right)=S\left(\boldsymbol{x}^{\prime}\right) \delta\left(\boldsymbol{x}^{\prime}-\boldsymbol{x}^{\prime \prime}\right),
$$

where $S\left(\boldsymbol{x}^{\prime}\right)$ is the spectral density of the sources and $*$ denotes complex conjugation. ${ }^{15}$ In the following, we refer to a wavefield that is generated by uncorrelated sources in the sense of equation (4) as a random wavefield. Using the assumption that the sources are uncorrelated, a representation theorem for the cross-correlation function between two pressure measurements $p\left(\boldsymbol{x}_{A}\right)$ and $p\left(\boldsymbol{x}_{B}\right)$ is given by

$$
C\left(\boldsymbol{x}_{A}, \boldsymbol{x}_{B}\right)=p\left(\boldsymbol{x}_{A}\right) p^{*}\left(\boldsymbol{x}_{B}\right)=\int_{D} G\left(\boldsymbol{x}_{A}, \boldsymbol{x}\right) G^{*}\left(\boldsymbol{x}_{B}, \boldsymbol{x}\right) S(\boldsymbol{x}) d \boldsymbol{x} .
$$

Here, the cross-correlation function $C\left(\boldsymbol{x}_{A}, \boldsymbol{x}_{B}\right)$ models the forward wavefield between two measurement locations. In equation (5), we use the fact that one domain integral can be dropped when the sources are uncorrelated. It has to be noted that the equality of the cross-correlation function to the convolution of the Green's functions with the sources solely holds for the forward wavefield from the sources to the receivers. Ultimately, we seek to determine the Green's function that describes the wavefield between these two measurement locations by using the cross-correlation function in equation (5). Hence, it needs to be shown that the cross-correlation function of pressure measurements from a random wavefield at two receiver positions is an approximation of the corresponding Green's function. Theoretical derivations proving this convergence ${ }^{15,18}$ are based on further simplifications that might be hard to fulfill in a real application. Sager et al. ${ }^{19}$ have shown that these assumptions may be dropped at the expense of higher computational costs and more complicated algorithm. As a proof of concept for random field interferometry on the medical scale, we first keep it simple and use the traditional approach of interferometry with Green's functions retrieval. Albeit the strong assumptions inherent to the traditional approach, experimental studies ranging from large-scale geophysical applications ${ }^{20}$ to small scale laboratory experiments ${ }^{21}$ have shown that the concept of using random field cross-correlations to extract times of flight is rather robust against violations of the theoretical assumptions. The relationship between the crosscorrelation functions of a random wavefield and the direct responses, given certain assumptions, can thus be formulated as

$$
G\left(\boldsymbol{x}_{A}, \boldsymbol{x}_{B}\right)-G^{*}\left(\boldsymbol{x}_{A}, \boldsymbol{x}_{B}\right) \propto i \omega C\left(\boldsymbol{x}_{A}, \boldsymbol{x}_{B}\right) .
$$

Compared to equation (5), the previous expression states that the cross-correlation function is proportional to the Green's function between the receivers $G\left(\boldsymbol{x}_{A}, \boldsymbol{x}_{B}\right)$ minus its time-reversed version $G^{*}\left(\boldsymbol{x}_{A}, \boldsymbol{x}_{B}\right)$. It is important to note that in the tomographic reconstruction as introduced in section 2.3 we use the time-domain cross-correlation function, which is readily obtained from applying the inverse Fourier transform. This implies that when working in the time domain, the time derivative of the cross-correlation function should be used. The time of flight between two receiver positions is then identified in the cross-correlation function by the largest sum of the amplitude products. Consequently, by computing the cross-correlation function between two random signals, we can directly approximate the corresponding Green's function. We note that all specific source influences have been eliminated in equation (6). More specifically, this means that in equation (6) no active sources are present, but the cross-correlation function is the signal which one receiver would record when the other is given an impulsive excitation. This is sometimes referred to as the virtual source principle, ${ }^{22,23}$ which implies that for example the receiver at position $\boldsymbol{x}_{A}$ records a signal as if receiver $\boldsymbol{x}_{B}$ was an impulsive source or vice versa.

An additional benefit of exploiting the relationship (6) is that the cross-correlation is not limited to first arrivals only, but comprises all travel paths between the two points. Thus multiple reflections and scattering events are as well present in the cross-correlation and could provide potentially more information on the medium.

\subsection{Travel time tomography}

Ultimately, we seek to reconstruct a numerical breast phantom from the cross-correlation times of flight by using a tomographic procedure. The time of flight or travel time between two receivers $t$ may be related to the speed of a wave along a ray path $\Gamma$ as

$$
t(\Gamma)=\int_{\Gamma} s(\boldsymbol{x}) d l
$$


where $d l$ is the differential distance along a ray and the parametrization $s(\boldsymbol{x})=c^{-1}(\boldsymbol{x})$ is termed the slowness at position $\boldsymbol{x}$. The travel time $t(\Gamma)$ depends on the ray path, which follows from Fermat's principle. The acoustic ray path itself also depends on the slowness distribution $s(\boldsymbol{x})$, establishing a nonlinear relationship between the travel time and the speed of sound of the tissue. A typical approach to handle the non-linearity of equation (7) is to linearize about some initial reference slowness model. This means that instead of solving for the absolute slowness value $s(\boldsymbol{x})$, one seeks corrections to an initial model that approximates the real distribution of the speed of sound in the tissue. ${ }^{24}$ Equation (7) is the basis for any ray-based tomography procedure, which is in essence concerned with inverting the line integral relationship and is therefore treated by solving an inverse problem.

\subsection{Inverse Problem}

Having a good estimate of the Green's function between two measurement points, we can reconstruct the slowness distribution $\mathbf{m}$, which is the reciprocal of the speed of sound. Measured data $\mathbf{d}_{\text {obs }}$ is related to the material parameters $\mathbf{m}$ by the forward problem as

$$
\mathbf{d}_{\mathrm{obs}}=\mathbf{F m} .
$$

In the synthetic study, we model the times of flight using straight rays between the emitters and the receivers, which yields a linear forward operator $\mathbf{F}$ that maps slowness $\mathbf{m}$ to travel times $\mathbf{d}_{\text {obs }}$. In general, one is interested in finding the model that best describes the observed data, which involves the inverse of the forward operator $\mathbf{F}^{-1}$ to the observed data $\mathbf{d}_{\mathrm{obs}}$ and is denoted as the inverse problem. Finding the inverse of the forward operator is often not trivial, therefore the model parameters are estimated by minimizing the regularized least-squares misfit functional

$$
\chi(\mathbf{m})=\frac{1}{2}\left\|\mathbf{F m}-\mathbf{d}_{\mathrm{obs}}\right\|^{2}+\frac{\alpha}{2} \mathbf{R}(\mathbf{m}),
$$

where $\mathbf{R}(\mathbf{m})$ is the regularization term and $\alpha>0$ controls the contribution of the regularization.

Instead of minimizing the misfit function (9) with respect to a homogeneous background model, it is common practice to minimize the misfit of the perturbations of the model parameters with regard to a homogeneous background model, thus $\mathbf{m}=\mathbf{m}_{\mathbf{0}}+\delta \mathbf{m}$. Due to the assumed linearity of $\mathbf{F}$, the forward problem can be rephrased to

$$
\mathbf{d}_{\mathrm{obs}}=\mathbf{F m}_{\mathbf{0}}+\mathbf{F} \delta \mathbf{m} .
$$

Note that if we use the parametrization $\mathbf{d}_{\mathrm{obs}}=\mathbf{d}_{\mathbf{0}}+\delta \mathbf{d}_{\mathrm{obs}}$ to describe the deviations of the measurements from the data that would be obtained from a homogeneous background, then $\mathbf{d}_{\mathbf{0}}=\mathbf{F m}_{\mathbf{0}}$ is the forward problem for the homogeneous background model and is readily computed. In the case of time-of-flight tomography, $\delta \mathbf{d}_{\text {obs }}$ are the travel time shifts between the cross-correlation travel times and the analytical solution for water. Minimizing the misfit functional with respect to the model parameter perturbations yields then

$$
\min _{\delta \mathbf{m}} \frac{1}{2}\left\|\mathbf{F} \delta \mathbf{m}-\delta \mathbf{d}_{\mathrm{obs}}\right\|^{2}+\frac{\alpha}{2} \mathbf{R}(\delta \mathbf{m}) .
$$

For the regularization term, we choose the spatial Hessian with respect to the perturbations of the model parameters, hence $\nabla^{2} \mathbf{R}(\delta \mathbf{m})=\nabla^{2}\|\delta \mathbf{m}\|_{2}^{2}$. This penalizes large oscillations in $\delta \mathbf{m}$ between neighboring cells and is referred to as second-order Tikhonov regularization ${ }^{25}$ or as ridge regression ${ }^{26}$ in statistical literature.

\section{RESULTS}

In this section, we present numerical results illustrating the proposed algorithm that extracts times of flight between any receiver positions in a USCT setup by cross-correlating recordings of an actively generated random wavefield and then uses this information to reconstruct a synthetic breast phantom. This section is split into two parts. In the first part, we present a proof of concept for the proposed method by benchmarking the reconstruction of a homogeneous background model with the attached speed of sound in water $(1500 \mathrm{~m} / \mathrm{s})$ to the analytical solution. In the second part, the tomographic reconstruction of the speed of sound distribution within the synthetic phantom using the Tikhonov regularization with the second derivative as introduced in the previous section is presented. We use a $2 \mathrm{D}$ numerical model of a horizontal slice in a ring-shaped aperture with 256 equiangular-spaced transducers being sources and receivers at the same time. The numerical experiments 
are performed using simulated wavefields with a central frequency of $1 \mathrm{MHz}$. The synthetic forward wavefield is generated by the modeling software suite Salvus, ${ }^{27}$ which implements the spectral-element method to solve the wave equation.

The numerical breast phantom considered for this study is shown in Figure 1. The phantom models a coronal slice through a breast with a diameter of $9.4 \mathrm{~cm}$, a high velocity skin layer shown in yellow and inclusions of various speeds of sounds modeling tissue regions with different parameters (e.g. due to malignant cell regions). The numerical domain is discretized on a rectilinear grid with node spacing of $1 \mathrm{~mm}$.

The justification of using cross-correlations in order to extract times of flight between receiver pairs depends on the assumption that the receivers record a wavefield that is generated by temporally uncorrelated sources. We denote such a wavefield as a random wavefield, honoring the fact that the sequence of individual source impulses, which are superimposed to form this wavefield, is completely random. In practice, this implies that all sources are activated randomly over a certain period of time and excite a wavefield that is equal to the superposition of all individual source wavefields. The amplitude of each source wavelet is chosen from a normal distribution to further increase the randomness. Then, the resulting wavefield is recorded at all receivers. The numerical domain is enclosed by reflecting boundaries simulating the tank walls. Reflections from the tank walls also promote the non-coherency in the wavefields. The reason for emphasizing the importance of the randomness in the wavefield stems from the fact that the crosscorrelation function between two receiver recordings only sums constructively at the expected lag times when a substantial number of waves pass through both

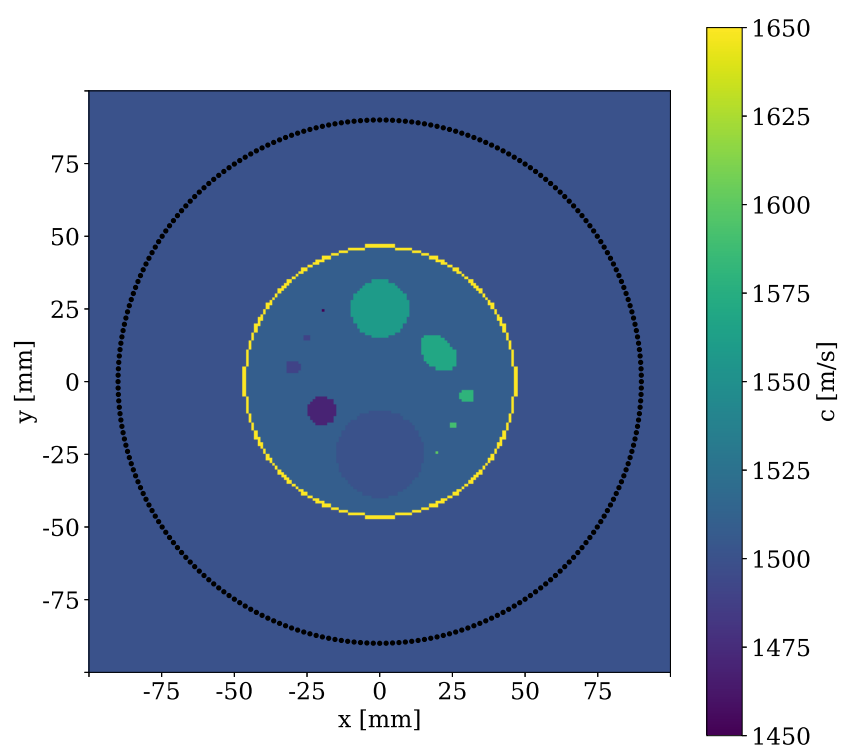

Figure 1: The experimental setup for the numerical study. The phantom is surrounded by 256 transducers, marked by $\cdot$. receivers and therefore sample the space in between them. These waves might be direct waves or reflections from the tank walls and internal inhomogeneities.

The accuracy of the cross-correlation travel times depends strongly on the quality of the Green's function approximation. Consequently, the main task of the cross-correlation procedure is to obtain a correlation function that displays distinct peaks at the time of flight between two receivers. At these peaks, the amplitudes of both recordings sum constructively. As outlined, this may not be a trivial task depending on the position of the receivers on the circular array with respect to each other. Figure 2 demonstrates the picking problem on two exemplary cross-correlation functions, where Figure 2a shows the result for a pair of receivers less than $16.2 \mathrm{~cm}$ apart (which corresponds to approximately $130^{\circ}$ between the two receivers on the circular array) and Figure $2 \mathrm{~b}$ displays a noisy cross-correlation for a pair more than $130^{\circ}$ apart. The maximum distance for two opposite receivers is $18 \mathrm{~cm}$. In the case of a smaller distance between the receivers, the recordings sum constructively at lag times that is equal to the time of flight between them. The peaks indicating the propagation time are clearly defined and easily picked. For larger distances though, the opposite is observed. In general, we can state that the further apart two receivers are, the more difficult it is to see features in the cross-correlation function that are related to the Green's function between these receivers. One explanation is that due to regular geometry of the setup, recordings from two opposite receivers are more coherent, thus the traces sum not only constructively at the expected lag times, but also at other times. Note that by definition, the cross-correlation function is symmetric. Positive time-lags are interpreted as Green's functions for signals from receiver A to B, and negative time lags correspond to signals from receiver B to A. Comparing the cross-correlation functions in Figure 2, we observe that this symmetry is broken. Analyzing the standard deviation between the positive and negative time 


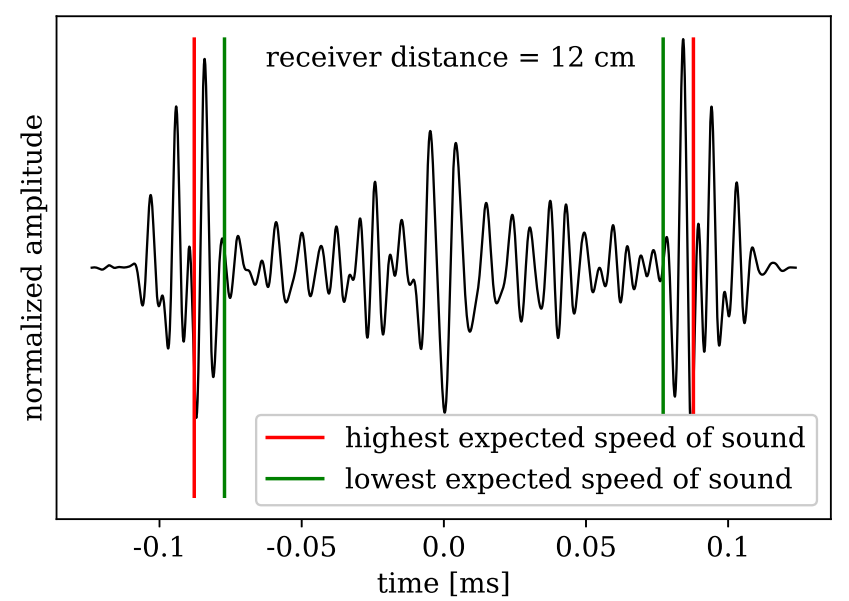

(a)

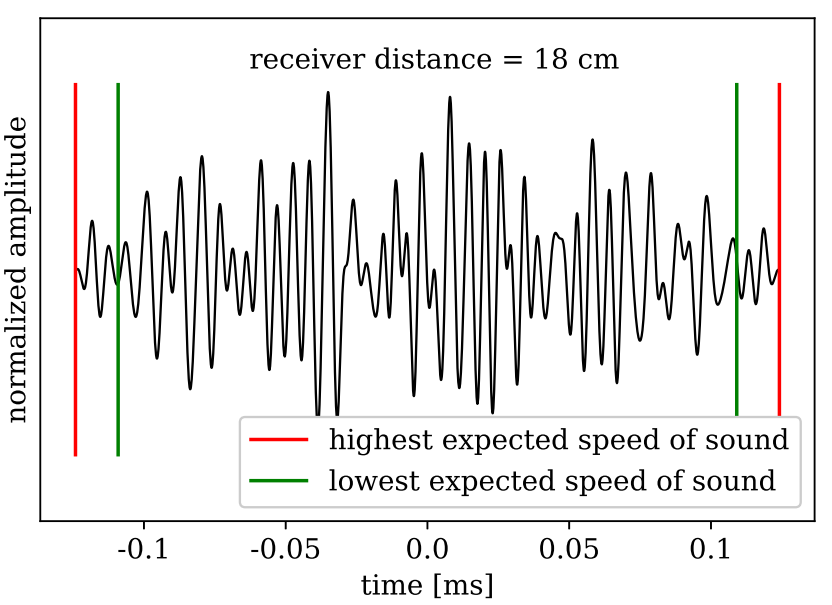

(b)

Figure 2: Two cross-correlation functions for different receiver pairs. Figure 2a shows an exemplary crosscorrelation of receivers that are positioned $12 \mathrm{~cm}$ apart, which corresponds to an angle of $90^{\circ}$ on the receiver array shown in Figure 1. Distinct peaks identify the empirical Green's function and indicate the time of flight. In contrast, the cross-correlation function for two opposite receivers in figure $2 \mathrm{~b}$ reveals the difficulty to select the peak associated to the time of flight for receivers further apart. Here, the distance between the receivers is $18 \mathrm{~cm}$ and corresponds to an angle of $180^{\circ}$. Form prior knowledge on lower and upper bounds to the speed of sound values within human tissue, a sliding picking window can be created, which helps to restrict valuable peaks within the cross-correlation function.

lags of the cross-correlation function can therefore be used as a measure of its convergence towards the Green's function. To address the picking difficulty, we make use of prior information on the speed of sound values within human breast tissue as provided by literature ${ }^{3}$ to constrain a picking window in which valid travel times for the concerned receiver pair are to be expected. This also allows us to differentiate between first arrivals from a virtual source to a receiver and peaks related to reflections from the boundary (as seen in Figure 2a, where the first peak indicates the Green's function and the second peak a boundary reflection).

To verify the algorithm, we attempt to reconstruct a 2D homogeneous background model simulating the numerical domain filled only with water. Knowing the receiver positions, the analytical solution for a homogeneous domain filled with water is readily calculated. On the basis of the travel time picks from the cross-correlation functions, we proceed to compute the travel time shifts with regard to the exact analytical solution. To evaluate the accuracy of the cross-correlation times of flight, we compare them to the analytical solution and calculate the difference, which will be referred to as the times of flight shifts in the following. For perfect data, the deviation between the picked travel times and the analytical solution should be zero. Realistically, we expect the picked travel times to be influenced by noise in the cross-correlations as well as errors in the picking process. This is observed in Figure 3, where the relative deviation in percent of the extracted cross-correlation times of flight from the analytical solution is displayed by the blue line. We show the relative difference for 150 receiver pairs, where one receiver acts as a virtual source and is recorded at all other receivers. Figure 3 shows the receiver pairs for the receiver located at 9 o'clock on the transducer array in Figure 1 being the virtual source. We chose to display only the relevant receiver pairs between locations at 11 o'clock and 7 o'clock, since for these pairs, the rays from the virtual source position at 9 o'clock start sampling the domain that will be influenced by the numerical phantom.

To examine the influence of speed of sound variations on the times of flight, we use a straight-ray inversion algorithm to calculate the expected times of flight for the phantom directly and compute the relative difference with respect to a homogeneous background model. When plotted, the relative difference between the phantom and the homogeneous background visualizes the effect of the speed of sound variations within the phantom shown 
by red line in figure 3. In order to use the cross-correlation times of flight to deduce speed of sound values, their relative error must be smaller than the effect of the speed of sound variations within the phantom on the times of flight. Figure 3 suggests that compared to the influence of the phantom, the relative error of the cross-correlation times of flight is sufficiently small, such that we expect the method to be able to detect speed of sound variations on the order of the numerical phantom correctly. For the benchmark test of a domain filled only with water, the extracted cross-correlation times of flight for the considered 150 receiver pairs differs by approximately $0.11 \%$ from the exact analytical solution. As seen in Figure 3, the effect of the speed of sound variations introduced by the phantom is relatively small. This stems from the fact that that although the mean speed of sound within the phantom deviates by approximately $3.5 \%$ from the speed of sound of water - thus the speed of sound is approximately $1550 \mathrm{~m} / \mathrm{s}$ - the overall influence of the phantom on the travel times is expected to be much lower. Since the phantom merely fills half of the domain, the rays spend a considerable time traveling through water. If we assume a ray to propagate through water for half its travel path, then the influence of the phantom accounts for a deviation of the time of flight of $1.7 \%$ compared to the analytical solution. This is reflected in the relative differences of the model compared to the homogeneous background solution seen in Figure 3.

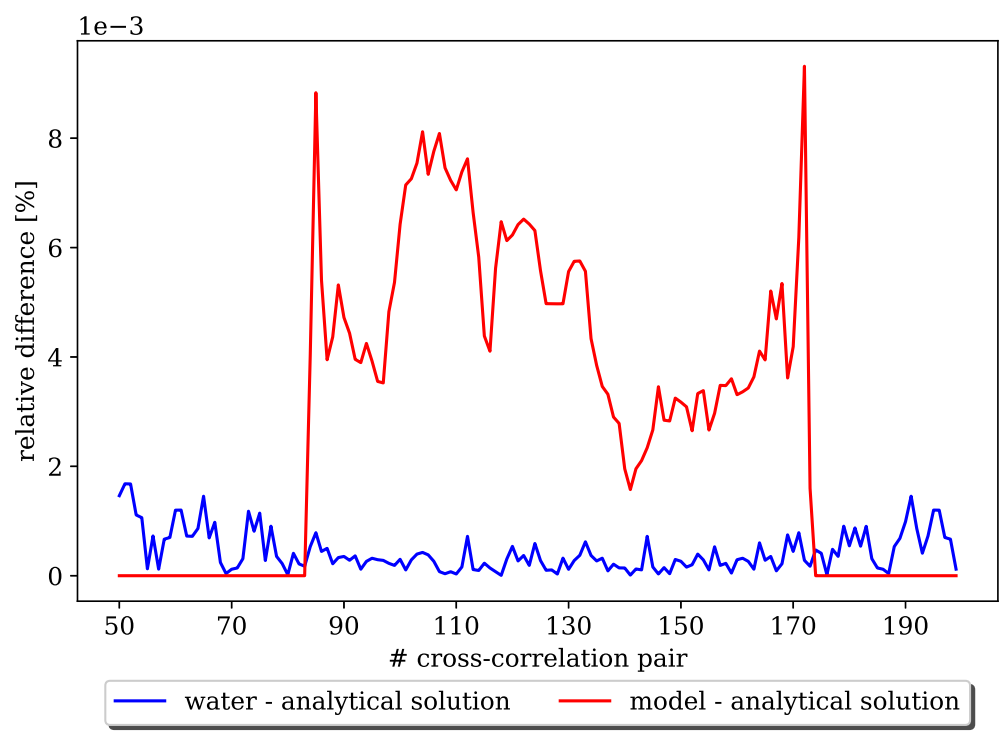

Figure 3: The benchmark test of the algorithm in order to reconstruct a homogeneous background model with a speed of sound of $1500 \mathrm{~m} / \mathrm{s}$ using 256 receivers and $1 \mathrm{MHz}$ central frequency in the forward simulation. The relative difference between the cross-correlation times of flight for water and the analytical solution for a homogeneous domain are shown in blue for the first 256 cross-correlation pairs, that is all the combinations for one receiver being a virtual source recorded at all other receivers. In red, the relative difference of the travel time data derived directly from the numerical model seen in Figure 1 with respect to a homogeneous background is shown.

To illustrate the potential of the proposed method to extract travel times that can be used as data in an image reconstruction algorithm, we finally attempt to reproduce the numerical phantom shown in Figure 1. Again, we use simulated data generated with a central frequency of $1 \mathrm{MHz}$, which is close to the order of frequencies that state-of-the-art scanning devices operate on. ${ }^{4,28,29}$

Figure $4 \mathrm{~b}$ displays the reconstruction result. When comparing to the ground truth, we note that the smallest recovered features are approximately $1 \mathrm{~cm}$ in diameter (see high velocity inclusion at 3 o'clock). The recovered speed of sound values are in the right range compared to the ground truth. The phantom imprint is clearly visible in the travel time shifts in Figure 4a, which visualizes all 256 cross-correlation pairs for one receiver acting as a virtual source. Note, that here we display the absolute times of flight shifts in contrast to Figure 3 where relative differences in percent are shown. For rays crossing only through water, the travel time shifts are close to zero as expected, whereas for rays that are tangent or crossing the phantom, the cross-correlation travel times deviate 


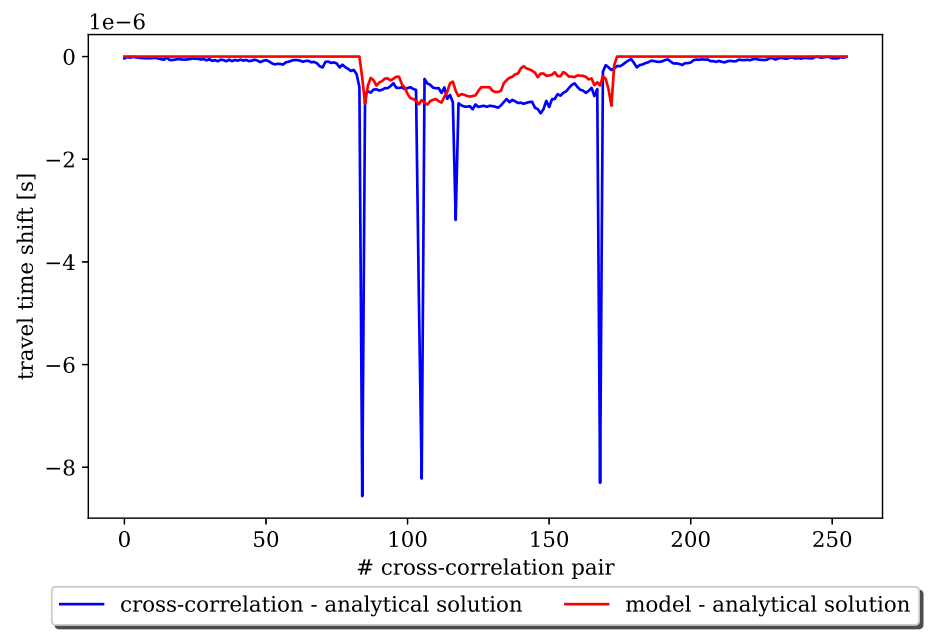

(a)

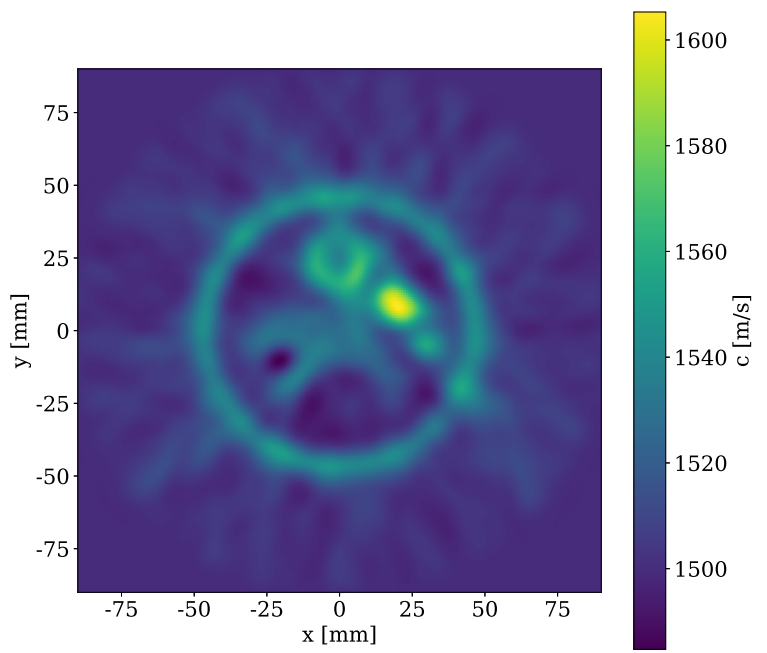

(b)

Figure 4: The travel time shifts between the analytical solution for a homogeneous domain with $c=1500 \mathrm{~m} / \mathrm{s}$ and the cross-correlation travel times (Figure 4a). For comparison, the travel time shifts between the analytical solution and the exact travel times, computed by using the numerical phantom in the straight-ray inversion (inverse crime), is shown in red. Figure $4 \mathrm{~b}$ shows the reconstruction for a setup with 256 sources and receivers and $1 \mathrm{MHz}$ central frequency used in the computation of the ground truth.

from the analytical solution. This can also be observed in the red curve, which shows the travel time shifts between the analytical solution and the travel times calculated directly from using the numerical phantom in the inversion. The total size of the phantom is slightly underestimated, which is a shortcoming of the straight-ray assumption.

\section{DISCUSSION}

With this work, we have shown a first numerical proof of concept for the extraction of times of flight from crosscorrelations of an actively generated wavefields on the scale of medical applications. The USCT setup could benefit from this new approach in several ways:

(i) individual source wavefields are substituted with one random wavefield;

(ii) the virtual source principle renders reference measurements to calibrate the sources redundant;

(iii) reflections from the tank walls, hitherto tried to be avoided, are incorporated in the extraction of time-offlight data.

To use one actively generated random wavefield instead of multiple individual source wavefields is especially interesting when considering the examination time needed for one patient. The shorter a single examination is, the higher the potential patient throughput could be, which means that more women could be screened in the same amount of time as for a current screening process.

A major challenge in traditional USCT acquisition methods is the need for calibration of the sources. This is a consequence of the aforementioned approach of calculating times of flight between receiver pairs by firing one source at a time and by recording the response at all receiver positions. Since the mechanical transducers do not have identical radiation pattern, the measurements will be influenced by the the source. More precisely this means that the electrical noise differs most likely for each transducer, which leads to systematic errors in the recorded first arrivals. To define the source characteristics, reference measurements with regard to a known medium (in the case of USCT this is water) are carried out. By retrieving the Green's function between two 
measurements, we obtain the impulse response and therefore we know the mechanism of the virtual source, which eliminates the need for reference measurements.

A critical aspect that defines whether the method has potential to be incorporated in a real-life application is its computational cost. In practice this means that the most important question to be answered is how long does it take to generate a random wavefield actively such that the cross-correlation function between individual recordings converges to the Green's function between two receiver positions. We have found that for the numerical study considered here, a simulation time of $15 \mathrm{~s}$ is sufficient to build up a wavefield with enough non-coherent information in the individual recordings. In vivo acquisition times for an entire USCT data set are reported to be currently on the order of minutes. ${ }^{4}$ For the clinical application, the data acquisition should be as short as possible to minimize effects due to movements of the patient or due to temperature variations of the water. Hence, the possibility to shorten the acquisition time is valuable. In addition to this, no rotation of the transducer array is needed to collect data from all angles when using a random wavefield.

Another challenge inherent to the USCT setup is that using conventional acquisition methods, reflections from the tank walls are undesired, but hard to avoid. The proposed random wavefield however incorporates these reflections and even benefits from the additional information. We have shown that one can extract the travel time between any pair of transducer positions from random wavefields by using cross-correlations and a stacking of A-scans.

In this work, we have only considered a non-attenuating medium for the purpose of illustrating the method. This is however far from being realistic. Incorporating attenuation mainly effects the generation of the random wavefield. If energy is lost too quickly, multiple reflection events are not recorded, thus the recorded wavefield is dominated by transmission data. When cross-correlating two recordings, this entails that the sum of amplitude products identifying the time of flight is not well defined anymore. In Figure 5, we examined the effect of attenuation on an exemplary cross-correlation function.

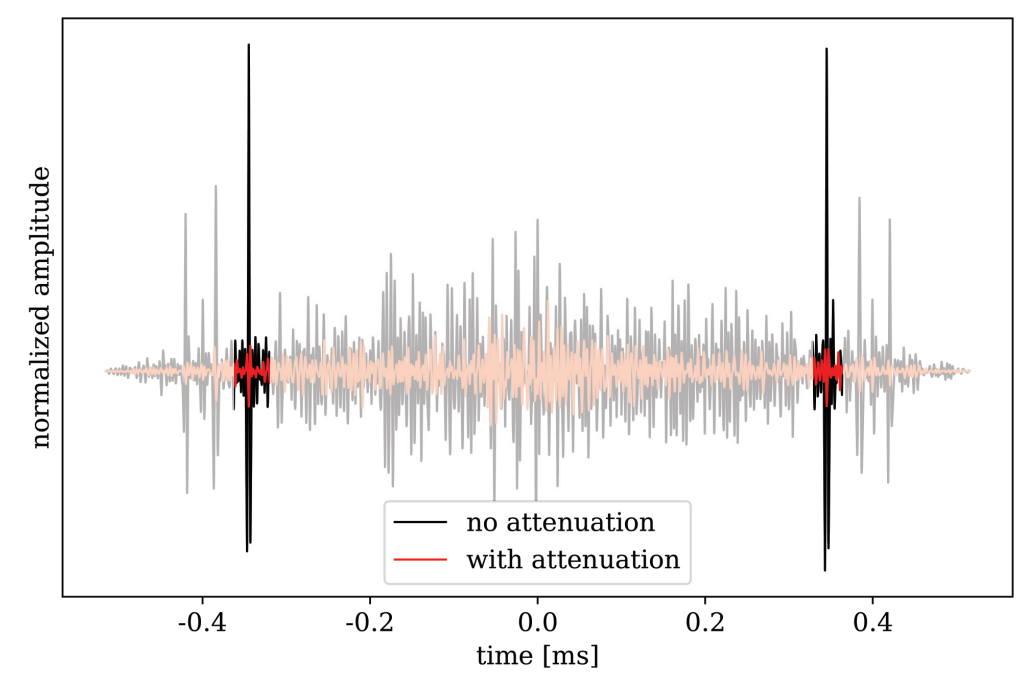

Figure 5: The effect of attenuation on the quality of the cross-correlation function for a single receiver pair. The traces have been normalized to the mean value of the cross-correlation functions respectively. We assume an attenuation coefficient of $\alpha=0.91$ or $Q=200$, which corresponds to values common in medical ultrasound. ${ }^{7}$ Amplitude losses in the waves due to the attenuation of the medium results in less information at later times. Peaks indicating the time of flight (non shaded area) are less distinct, while peaks in the center of the crosscorrelation are amplified.

The empirical Green's function is clearly identified by peaks when attenuation is neglected in the forward simulation. Multiplying the amplitude of the forward wavefield with an attenuation factor results in less prominent peaks at the time of flight. As an attenuation factor we used $\exp \left[-\frac{\omega t}{2 Q}\right],{ }^{16}$ where $Q=200$ is the temporal seismic attenuation coefficient. The latter can be converted to the attenuation factor $\alpha$ common in medical 
ultrasound as presented by Pratt et al. ${ }^{7}$ An attenuation coefficient of $Q=200$ corresponds to $\alpha=0.91$. Figure 5 suggests that although energy is diffused faster when attenuation is present, the time of flight could still be picked using the sliding window. Using more advanced processing tools that are based on the phase coherence of the recordings rather than their amplitude similarity at lag time may improve the quality of the cross-correlation function. ${ }^{30}$

Although the numerical study presented herein is carried out in $2 \mathrm{D}$, the extension to $3 \mathrm{D}$ should be possible. Future work will focus on improving the reconstruction algorithm, for instance by applying a more sophisticated regularization term such as total variation regularization. Exploiting the full potential of the retrieved Green's functions, the data coverage may be increased relative to the standard measurements of the USCT aperture. Beyond that, quantifying the effect of attenuation on the cross-correlation function is important when approaching real data. As the quality of the approximation of the Green's function depends on the random wavefield, improving the generation of the latter could result in cross-correlation functions with better identifiable peaks at the time of flight. Lastly, the limitations of the straight-ray approach in the inversion procedure could be overcome by finite frequency tomography and bent ray approximations. It is worthwhile noting that when extracting times of flight via the cross-correlation function, we do not introduce any assumptions on the nature of the ray. Thus the implementation of more sophisticated reconstruction algorithms is possible. There have been successful attempts that apply waveform tomography to medical imaging ${ }^{8,31-33}$ which generally leads to reconstructions of higher resolution although at higher computational costs. We use straight-ray tomography since it offers a trade-off between images of superior resolution and higher computational costs and is still the most widely applied tomographic approximation in medical imaging.

\section{CONCLUSIONS}

This work provides the basis for a framework that applies random field interferometry to ultrasound computed tomography. On the basis of a quasi-random wavefield, generated by a combination of reflection and transmission data, time of flight information is obtained by cross-correlation. Travel time shifts with regard to the analytical solution can be subsequently used to invert for a homogeneous background medium as well as for a breast phantom, rendering additional measurements to calibrate the sources redundant. The synthetic study retrieved the speed of sound distribution of the synthetic phantom well and highlight the potential for future applications.

\section{ACKNOWLEDGEMENTS}

The authors wish to thank Naiara Korta Martiartu and Daniel Bowden for fruitful discussions revolving around various topics covered in this paper. We gratefully acknowledge support by the Swiss National Supercomputing Centre (CSCS) under project grant s919.

\section{REFERENCES}

[1] Foster, F., Strban, M., and Austin, G., "The ultrasound macroscope: Initial studies of breast tissue," Ultrasonic Imaging 6(3), 243 - 261 (1984).

[2] Greenleaf, J. F., Johnson, S. A., and Bahn, R. C., "Quantitative cross-sectional imaging of ultrasound parameters," in [1977 Ultrasonics Symposium], 989-995 (1977).

[3] Greenleaf, J. F. and Bahn, R. C., "Clinical imaging with transmissive ultrasonic computerized tomography," IEEE Transactions on Biomedical Engineering BME-28(2), 177-185 (1981).

[4] Gemmeke, H., Hopp, T., Zapf, M., Kaiser, C., and Ruiter, N. V., "3d ultrasound computer tomography: Hardware setup, reconstruction methods and first clinical results," Nuclear Instruments and Methods in Physics Research Section A: Accelerators, Spectrometers, Detectors and Associated Equipment 873, 59 - 65 (2017). Imaging 2016.

[5] Sandhu, G., Li, C., Roy, O., Schmidt, S., and Duric, N., "Frequency domain ultrasound waveform tomography: breast imaging using a ring transducer," Physics in Medicine \& Biology 60(14) (2015).

[6] Malik, B., Terry, R., Wiskin, J., and Lenox, M., "Quantitative transmission ultrasound tomography: imaging and performance characteristics," Medical Physics 45 (05 2018). 
[7] Pratt, R., "Medical ultrasound tomography: lessons from exploration geophysics," Proceedings of the 1st International Workshop on Medical Ultrasound Tomography (01 2017).

[8] Pratt, R., Huang, L., Duric, N., and Littrup, P., "Sound-speed and attenuation imaging of breast tissue using waveform tomography of transmission ultrasound data," Proceedings of SPIE - The International Society for Optical Engineering 6510 (03 2007).

[9] Guasch, L., Calderón Agudo, O., Tang, M.-X., Nachev, P., and Warner, M., "Full-waveform inversion imaging of the human brain," bioRxiv (2019).

[10] Fincke, J. R., Feigin, M., Prieto, G. A., Zhang, X., and Anthony, B., "Towards ultrasound travel time tomography for quantifying human limb geometry and material properties," in [Medical Imaging 2016: Ultrasonic Imaging and Tomography], 9790, 470 - 480, International Society for Optics and Photonics, SPIE (2016).

[11] Sabra, K. G., Conti, S., Roux, P., and Kuperman, W. A., "Passive in vivo elastography from skeletal muscle noise," Applied Physics Letters 90(19), 194101 (2007).

[12] Gallot, T., Catheline, S., Roux, P., Brum, J., Benech, N., and Negriera, C., "Passive elastography: Shearwave tomography from physiological-noise correlation in soft tissues," Ultrasonics, Ferroelectrics and Frequency Control, IEEE Transactions on 58, 1122 - 1126 (07 2011).

[13] Shapiro, N. M., Campillo, M., Stehly, L., and Ritzwoller, M. H., "High-resolution surface-wave tomography from ambient seismic noise," $\mathbf{3 0 7}(5715), 1615-1618$ (2005).

[14] Saygin, E. and Kennett, B. L. N., "Crustal structure of australia from ambient seismic noise tomography," Journal of Geophysical Research: Solid Earth 117(B1) (2012).

[15] Wapenaar, K. and Fokkema, J., "Green's function representations for seismic interferometry," Geophysics 4(71) (2006).

[16] Aki, K. and Richards, P., [Quantitative Seismology], Geology Seismology, University Science Books (2002).

[17] Fichtner, A., Nakata, N., and Gualteri, L., [Seismic ambient noise], Cambridge University Press (2019).

[18] Weaver, R. L. and Lobkins, O. I., "Elastic wave thermal fluctuations, ultrasonic waveforms by correlation of thermal phonons," The Journal of the Acoustical Society of America (113), 2611 (2003).

[19] Sager, K., Ermert, L., Boehm, C., and Fichtner, A., "Towards full waveform ambient noise inversion," Geophysical Journal International 212, 566-590 (10 2017).

[20] Gerstoft, P., Sabra, K. G., Roux, P., Kuperman, W. A., and Fehler, M. C., "Green's functions extraction and surface-wave tomography from microseisms in southern california," GEOPHYSICS 71(4), SI23-SI31 (2006).

[21] Malcolm, A., Scales, J., and Tiggelen, B., "Extracting the green function from diffuse, equipartitioned waves," Physical review. E, Statistical, nonlinear, and soft matter physics 70, 015601 (02 2004).

[22] Wapenaar, C., Slob, E., Snieder, R., and Curtis, A., "Tutorial on seismic interferometry: Part 2 - underlying theory and new advances," Geophysics 5(75) (2010).

[23] Bakulin, A. and Calvert, R., "The virtual source method: Theory and case study," Geophysics 71(4), SI139-SI150 (2006).

[24] Bording, R. P., Gersztenkorn, A., Lines, L. R., Scales, J. A., and Treitel, S., "Applications of seismic travel-time tomography," Geophysical Journal International 90, 285-303 (08 1987).

[25] Golub, G. H., Hansen, P. C., and O'Leary, D. P., "Tikhonov regularization and total least squares," SIAM Journal on Matrix Analysis and Applications 21(1), 185-194 (1999).

[26] Johansen, T. A., "On tikhonov regularization, bias and variance in nonlinear system identification," Automatica 33(3), 441 - 446 (1997).

[27] Afanasiev, M., Boehm, C., van Driel, M., Krischer, L., Rietmann, M., May, D. A., Knepley, M. G., and Fichtner, A., "Modular and flexible spectral-element waveform modelling in two and three dimensions," Geophysical Journal International 216, 1675-1692 (11 2018).

[28] Duric, N., Littrup, P., Li, C., Roy, O., Schmidt, S., Cheng, X., Seamans, J., Wallen, A., and Bey-Knight, L., "Breast imaging with SoftVue: initial clinical evaluation," in [Medical Imaging 2014: Ultrasonic Imaging and Tomography], Bosch, J. G. and Doyley, M. M., eds., 9040, 208 - 215, International Society for Optics and Photonics, SPIE (2014). 
[29] Wiskin, J., Borup, D., Johnson, S., Andre, M., Greenleaf, J., Parisky, Y., and Klock, J., "Three-dimensional nonlinear inverse scattering: Quantitative transmission algorithms, refraction corrected reflection, scanner design and clinical results," Proceedings of Meetings on Acoustics 19(1), 075001 (2013).

[30] Schimmel, M., Stutzmann, E., and Gallart, J., "Using instantaneous phase coherence for signal extraction from ambient noise data at a local to a global scale," Geophysical Journal International 184(1), 494-506 (2011).

[31] Wang, K., Matthews, T., Anis, F., Li, C., Duric, N., and Anastasio, M., "Waveform inversion with source encoding for breast sound speed reconstruction in ultrasound computed tomography," 62 (12 2014).

[32] Boehm, C., Martiartu, N. K., Vinard, N., Balic, I. J., and Fichtner, A., "Time-domain spectral-element ultrasound waveform tomography using a stochastic quasi-Newton method," in [Medical Imaging 2018: Ultrasonic Imaging and Tomography], Duric, N. and Byram, B. C., eds., 10580, 92 - 100, International Society for Optics and Photonics, SPIE (2018).

[33] Agudo, O. C., Guasch, L., Huthwaite, P., and Warner, M., "3D imaging of the breast using full-waveform inversion," in [Proceedings of the International Workshop on Medical Ultrasound Tomography: 1.-3. Nov. 2017], 99, KIT Scientific Publishing (2018). 